\title{
Comprehensive pharmaceutical care to prevent drug-related readmissions of dependent-living elderly patients: a randomized controlled trial
}

R. Lenssen ${ }^{1,7,8}$, K. Schmitz ${ }^{1,7}$, C. Griesel ${ }^{1}$, A. Heidenreich ${ }^{2,9}$, J. B. Schulz ${ }^{3}$, C. Trautwein ${ }^{4}$, N. Marx ${ }^{5}$, C. Fitzner ${ }^{6}$, U. Jaehde ${ }^{7}$ and A. Eisert ${ }^{1 *}$

\begin{abstract}
Background: Elderly patients are vulnerable to adverse drug reactions (ADRs). Drug-related readmissions (DRRs) can be a major consequence of ADR. Therefore, this study aimed to investigate the effects of a ward-based, comprehensive pharmaceutical care service on the occurrence of DRRs as the endpoint in dependent-living elderly patients.

Methods: A randomized, controlled trial was performed at a German University Hospital. Patients fulfilling the following criteria were eligible: admission to a cooperating ward, existing drug therapy at admission, 65 years of age and older, home-care or nursing home residents in ambulatory care, and a minimum hospital stay of three days. Patients received either standard care (control group) or pharmaceutical care (intervention group). Follow-up consultations were conducted for each patient at 1, 8, 26, and 52 weeks after discharge. The time to DRR was defined as the primary outcome measure and was analysed using the log-rank test. The Cox-proportional hazard model was used for risk factor analysis.

Results: Sixty patients ( $n=31$ intervention group, $n=29$ control group) participated in the study. For patients in the intervention group, the median time to DRR was prolonged; however, the level of statistical significance was not reached (log-rank test $P=0.068 ; \mathrm{HR}=3.28, P=0.086$ ). When the risk factors 'age' or 'length of stay on the ward' were added to the Cox proportional hazard model, patients in the control group exhibited a significantly higher risk of experiencing a DRR than patients of the intervention group ( $H R=4.62 ; P=0.028$ including age and $H R=5.76 ; P=0.033$ including length of stay on the ward).

Conclusions: Our findings demonstrate the successful implementation of ward-based, comprehensive pharmaceutical care for dependent-living elderly. Despite a low participation rate, which led to an underpowered study, the results provide a preliminary efficacy signal and effect size estimates to power a definitive trial.
\end{abstract}

Trial registration: Clinicaltrials.gov identifier: NCT01578525, prospectively registered April 13, 2012.

Keywords: Adverse drug reactions, Drug-related readmissions, Elderly patients, Pharmaceutical care

\footnotetext{
* Correspondence: aeisert@ukaachen.de

${ }^{1}$ Hospital Pharmacy, University Hospital RWTH Aachen, Steinbergweg 20,

52074 Aachen, Germany

Full list of author information is available at the end of the article
}

(c) The Author(s). 2018 Open Access This article is distributed under the terms of the Creative Commons Attribution 4.0 International License (http://creativecommons.org/licenses/by/4.0/), which permits unrestricted use, distribution, and reproduction in any medium, provided you give appropriate credit to the original author(s) and the source, provide a link to the Creative Commons license, and indicate if changes were made. The Creative Commons Public Domain Dedication waiver (http://creativecommons.org/publicdomain/zero/1.0/) applies to the data made available in this article, unless otherwise stated. 


\section{Background}

Drug-related adverse events have been found to be the most common type of adverse events in hospitals and after discharge [1]. Moreover, up to $30 \%$ of all hospital admissions of elderly patients are drug-related [2, 3]. Therefore, strategies to prevent adverse drug reactions (ADRs) and drug-related hospital admissions are urgently needed, particularly for elderly patients [4-6]. In addition to other medication safety initiatives, national and international organizations recommend including pharmacists on health care teams to improve medication safety [7-11].

Several studies have demonstrated the positive patientindividual and economic impacts of pharmaceutical care [12-16]. Despite these results, few studies have evaluated the efficacy of pharmaceutical care services in hospitalized patients to reduce patient morbidity and mortality, particularly in high-quality and long follow-up trials [6]. Pharmaceutical care services are still limited and underrepresented in many countries in hospital as well as ambulatory care. The provision of those services, may prevent adverse drug reactions and subsequent hospital admissions. Therefore, the effect of a ward-based pharmaceutical care service on drug-related readmissions is a relevant and interesting topic for research $[13,17,18]$.

Age and number of drugs have been found to be risk factors for $\operatorname{ADR}[19,20]$. Furthermore impaired cognition and a dependent-living situation are known risk factors [19, 21], which implies that elderly patients in dependent-living situations in ambulatory care, such as 'being home-cared' or 'living in nursing homes', are a vulnerable group for drug-related problems and ADRs. Moreover, those patient groups are often excluded in clinical trials, even in studies focusing on high-risk populations and elderly persons [7]. To the best of our knowledge, this study is the first to evaluate the effect of a ward-based, comprehensive pharmaceutical care service in dependentliving elderly patients and its potential to reduce the time until drug-related readmissions (DRRs).

\section{Methods}

The study was set up as a randomized, controlled trial and conducted between April 2012 and March 2014, including a one-year recruitment and a one-year follow-up for each patient. The study was approved by the local ethics committee and is registered at clinicaltrials.gov.

\section{Participants}

Patients 65 years of age and older, who were home-cared or nursing home residents in ambulatory care, admitted to one of the cooperating wards with a minimum hospital stay of expected three days and existing medication at admission were eligible for this study. Cooperation wards were four departments of non-intensive care units at the
German University Hospital Aachen, Germany: the Departments of Urology, Neurology, Internal Medicine III (Gastroenterology and Metabolic Disorders) and Internal Medicine I (Cardiology, Pneumology and Angiology). We started recruitment with the three departments of the previous study (Urology, Neurology and Internal Medicine III). Because of the low participation rate, we added the department of Internal Medicine I after 6 months. Home-cared participants could receive care from informal or formal care-givers (e.g., family members or nurses). Informed consent was obtained from all individual participants or their legal representatives. After providing their consent, patients were randomized either to standard care (control group) or comprehensive pharmaceutical care (intervention group). Patients were randomized successively after being included in the study. Block randomization was chosen with 10 participants per block. A randomization list was generated at www.randomization.com. After study enrolment, the current number of included patient was the indicator for the allocation on the randomization list. Enrolment and allocation to the study group was conducted by the researcher (RL). The intervention was conducted by clinical pharmacists of the University hospital pharmacy (KS, CG, NH). Previous participation in the study was defined as the exclusion criterion.

\section{Intervention}

The comprehensive pharmaceutical care service was established in a previous study and included a detailed medication history, medication reconciliation and a medication safety check directly after inclusion in the study and during the entire stay on the cooperating wards [20]. Medication was checked for new prescriptions, and a medication review was repeated with each newly prescribed drug. Medication safety checks included the plausibility of medication, check for drug allergies, renal/liver dysfunction and dosage adjustment, relevant laboratory data, contraindications, dosage, drugdrug interactions, adverse drug reactions, medications before surgery or other interventions, adequate duration of drug therapy, need for patient information, and therapeutic drug monitoring. Transitional care at discharge included medication reconciliation at discharge and providing recommendations for the discharge letter. After discharge, the comprehensive pharmaceutical care service ended, and all study patients received their 'standard care'. The pharmacists had access to the data needed for the pharmaceutical care service. The service included also face-to-face counselling with the patients (e.g., for performing a detailed medication history or clarify potential problems in drug therapy).

Drug-related problems (DRPs) were identified during the entire pharmaceutical care process during the hospital 
stay. For each DRP, a specific recommendation was addressed to the health care team. Each recommendation was discussed with the healthcare team, and the physician decided if he or she followed the recommendations. The medication was checked again and registered, if the recommendation was partly or fully followed. The DRPs were documented and classified using the APS-Doc system [22], which we extended with three more subcategories in 'others'.

All pharmaceutical care activities were defined in standard operating procedures and performed by independent, trained clinical pharmacists to ensure the same standard of intervention for all patients in the intervention group. To ensure the equality of the observations for both treatment groups, intervention pharmacists (CG, KS, NH) worked independently from the researcher (RL). The intervention pharmacists were trained in all details of the SOP. They had one to three years of experience in clinical pharmacy. The intervention was established in three departments for the duration of the previous study. After closing that study, the intervention was not transferred to daily routine. Thus, standard care did not include clinical pharmacists in the health care team on the ward. Clinical pharmacists in this hospital are working in the hospital pharmacy and have contact with the wards mainly by telephone. A detailed medication review was only performed on explicit request of a physician. As described in our previous study, the routine medication process on the ward was performed by physicians and nurses.

\section{Data collection}

For the control and intervention groups, a researcher collected all relevant information on case report forms (CRF), designed for this study, including the current medication on the ward and demographic data, such as age, gender, social status (home-cared, nursing home resident), laboratory data and diagnoses. During the entire stay on the ward, ADR-suspicious symptoms were detected and discussed with the physician if necessary.

After discharge from the cooperating wards patients were contacted as pre-defined in the study protocol 1, 8, 26 and 52 weeks after discharge. Follow-up visits were conducted as semi-structured interviews, preferably with an interview guide, with the patient or responsible relatives or nurses who cared for the patient. Within the interviews current medication, ADR-suspicious symptoms and hospital readmissions to any hospital that occurred during the time after the last interview were documented. Additionally, available laboratory data, discharge letters and other patient documentation were examined.

Medication changes were determined from discharge medication to medication of the follow-up point. Changes in the strength, dose, dosage, dosing regimen or drug were included.

\section{Sample size estimation}

Based on the previous results of Gillespie et al., an $80 \%$ reduction in drug-related readmissions was assumed. Previous own results on the cooperating wards showed 2.6 DRPs per patient 65 years and older [20]. This DRP rate was consistent with the results of Gillespie et al. (2.6 DRPs/patient), despite their older population (80 years and older) [13]. In addition, the following parameters were set: drop-out rate $25 \%$, power $80 \%$, significance level $\alpha$ 0.05, and two-sided log rank test. The recruitment period was set at 12 months, with a follow-up period of 12 months for each patient. A sample size of 278 patients (139 patients and 15 events per group) was calculated using nQuery Advisor ${ }^{\oplus}$ 7.0, Statistical Solutions Ltd., Cork, Ireland. Therefore, the sample size was set to 300 patients.

\section{Outcome measures}

The primary endpoint was the occurrence of drugrelated readmissions (DRRs), measured over one year at four pre-defined contact times after discharge. DRR was defined as re-hospitalization of a discharged patient due to an adverse drug reaction (ADR) in any hospital. ADRsuspicious symptoms were defined as symptoms occurring in plausible context of medication use including inherent ADRs and ADRs due to medication errors. ADR-suspicious symptoms also included changes in laboratory data in a clinically relevant manner. All ADR-suspicious symptoms reported at the follow-up consultation were documented. It was investigated and documented when the symptoms occurred.

All ADR-suspicious symptoms and hospital readmissions were reviewed in a two-step process after data collection period. First, three pharmacists assessed all cases of ADR-suspicious symptoms and hospital readmissions ( $n=157)$; they were blinded regarding the allocation of the patients to the control or intervention group. The ADR-suspicious symptoms were classified as 'no ADR', 'potential ADR' or 'ADR' using the causality criteria by Arimone et al. [23]. In a second step, all 'potential ADR' $(n=34)$ were again assessed by three independent experts (community pharmacist, hospital pharmacist, physician). A majority decision was made after the experts voted on a scale as described by Arimone et al. [23]. Potential ADRs were thereby classified in seven causality levels: 'ruled out,' 'unlikely,' 'doubtful,' 'indeterminate,' 'plausible,' 'likely,'certain'. The causality levels 'certain', 'likely' and 'plausible' were regarded as ADR. Preventability and ameliorability were assessed according to Schumock and Thornton [24]. All preventable/ameliorable ADRs were categorized, according to their severity using the NCCMERP criteria [25].

In our study, ADR was defined as a harmful and unintended reaction on a medical product, which can occur 
under conditions of intended use or due to a medication error [26].

Moreover, as secondary endpoints, adverse drug reactions, potentially inappropriate medication (PIM) using the PRISCUS list [27] and the number of changes in medication after discharge were documented. In the intervention group, the number of drug-related problems and the number of accepted recommendations were assessed as further endpoints.

A drug-related problem (DRP) was defined as an "event or circumstance involving drug therapy that actually or potentially interferes with desired health outcomes", as stated by the Pharmaceutical Care Network Europe [28].

\section{Data analysis}

Data from CRFs were entered in Microsoft Excel ${ }^{\circ}$ (Microsoft Corporation, Redmond, WA, USA) spreadsheets for analysis. Statistical analysis was performed using SAS ${ }^{\circ}$ 9.1.3 (SAS Institute Inc., Cary, NC, USA). DRRs were analysed using the log-rank test to compare the time until occurrence of DRR. To consider potential risk factors on DRR Cox proportional hazard models were generated including 'treatment group' and one of the above-mentioned risk factors as covariates. The risk factors 'gender' and 'living situation' were included as binary variables, all other risk factors ('age,' "length of stay on the ward,' 'number of PIM drugs', 'number of changes in medication after discharge,' 'number of drugs during stay on the ward') were included as continuous variables. Hazard ratios (HR) were calculated to express the risk ratio for DRRs between the treatment groups [29].

To compare the baseline data in both study groups we used Satterthwaite t-test for 'age', length of hospital stay' and 'number of drugs during hospital stay'. For the parameters 'gender' and 'living situation', we used the Fisher exact test.

\section{Results}

During the one-year recruitment period, 61 patients were eligible and willing to participate (participation rate $17.7 \%)$. The number of recruited patients in each department is shown in Appendix 1. Thirty patients were allocated to the control group and 31 patients to the intervention group. One patient in the control group wished to be excluded from the study soon after randomization. Hence, 60 patients were analysed (for the study flow chart, see Fig. 1) after the previously defined one-year follow-up for each patient ended. Forty patients completed the follow-up. The inability to reach a patient (e.g., the patient moved to new address or died) caused censoring. The details are listed in Appendix 2.

The mean age of the participants was $77.6 \pm 7.9$ years; $60 \%$ of the participants were female, and $70 \%$ of participants were home-cared. On average, the participants had $16.8 \pm 6.9$ drugs in their medication on the wards (see Table 1). Five patients received no pharmaceutical care consultation because they were discharged before counselling. We performed an intention-to-treat analysis and included these data in the analysis.

Of the 30 patients in the intervention group, 26 received pharmaceutical care (see Fig. 1), and 100 DRPs were detected. Of them, $72 \%$ were partly or fully solved by the healthcare team in cooperation with the pharmacists. The detailed APS-Doc categories are shown in Appendix 3.

The mean time for providing pharmaceutical care during the initial hospital stay was $5.9 \mathrm{~h}$ per patient.

\section{Time to drug-related readmission}

Regarding the one-year follow-up in total, the median time until onset of DRR in the intervention group was longer compared to the control group. In the logrank test, however, this difference was not significant $(p=0.068$, see Fig. 2). Characteristics of the curves showed rare and later DRRs in the intervention group than in the control care group. The risk for DRR was higher under standard care compared to comprehensive pharmaceutical care (HR: 3.276 [CI: 0.844-12.716]; $p=0.0864$ ). However, no statistical significance was shown.

\section{Preventable drug-related readmissions}

In total, thirteen DRRs were documented during the 12-month follow-up (see Table 1). Seven patients in the control group (CG) and three patients in the intervention group (IG) exhibited at least one DRR in the follow-up period (see Table 1).

Thirty-five ADRs were observed during follow-up period. 14 ADRs (40\%) were judged to be preventable or ameliorable and categorized according to NCC-MERP for severity. Preventable or ameliorable ADRs of category F-H requiring a hospital stay can be regarded as preventable DRRs. Four preventable DRRs occurred in the control group and one in the intervention group (see Table 1).

Among the 60 patients, we measured 52 readmissions to any hospital during the follow-up period. Of them, 13 readmissions were judged to be drug-related readmissions in 10 patients, as shown in Table 1. These drug-related readmissions $(n=13)$ caused a mean stay of $19.8 \pm 19.1$ days. Preventable DRRs $(n=5)$ caused a mean stay of $13.4 \pm 30.7$ days.

\section{Risk factors for drug-related readmissions}

A detailed risk factor analysis was performed based on Cox proportional hazard models, including the potential covariates age, gender, living situation, length of stay on the ward, number of drugs on the ward, number of PIM using the PRISCUS list [27] and number of changes in medication after discharge (see Table 2). Age, the length 
Fulfilled inclusion criteria $(n=345)$

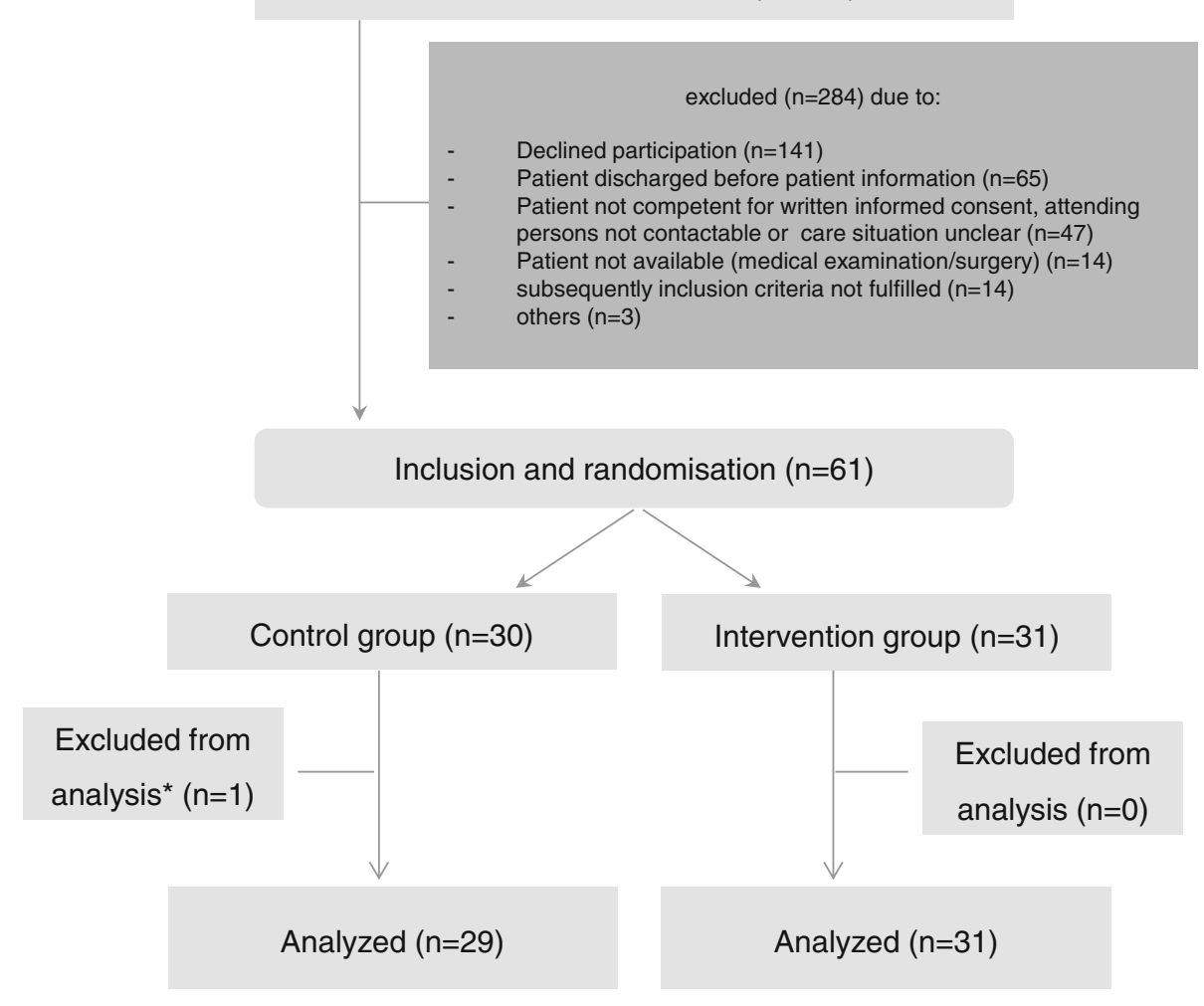

${ }^{*}$ Patient was randomized, informed consent was retracted soon after randomization

Fig. 1 Patient flow chart

of stay on the ward, and the number of changes in medication after discharge were found to be statistically significant risk factors for DRR.

An older age was associated with a lower risk of DRR $(\mathrm{HR}=0.86 ; \mathrm{CI}: 0.76-0.97 ; P<0.05)$. Including age in the univariate Cox proportional hazard model, the control group showed an approximately five-fold risk for DRR compared to the intervention group $(\mathrm{HR}=4.62$; CI: $1.18-18.13 ; P<0.05)$.

Each day the hospital stay was prolonged, the risk of a DRR increased by 1.1 -fold $(\mathrm{HR}=1.10 ; \mathrm{CI}$ : 1.01-1.19; $P<0.05)$. A longer stay of one week resulted in a two-fold risk increase $(\mathrm{HR}=1.91 ; \mathrm{CI}: 1.11-3.3 ; P<0.05)$. Including this risk factor in the univariate model, the control group had an approximately six-fold risk for DRR compared to the intervention group $(\mathrm{HR}=5.76$; $\mathrm{CI}$ : $1.15-28.85 ; P<0.05)$.

Although the number of changes in medication was found as a risk factor on DRR, the treatment group had no significant effect on DRR when including this risk factor.

For the time to ADR during follow-up in the intervention group compared with the control group, the log-rank test $(p=0.0684)$ and hazard ratio (HR:0.305;
CI: $0.079-1.185 ; \quad p=0.0864)$ showed no statistical significance.

\section{Discussion}

This study showed a potential positive impact of a comprehensive pharmaceutical care service and its potential to increase the time until DRR after discharge of elderly, cared patients in dependent-living situations. In the control group, there was a tendency for a higher risk for DRR compared to the intervention group receiving pharmaceutical care. However, the hazard ratio did not reach statistical significance, likely due to the low number of events.

The low participation rate of $17.7 \%$ must be considered when interpreting the results. The possible reasons for the non-participation were high age and morbidity of the patients. In our study, $41 \%$ of the patients declined to participate. Another study, with a decline rate of $36 \%$, showed similar factors (e.g., age, gender, morbidity) for non-participation in ambulatory care [30]. Home-care patients and nursing home residents are often excluded from other studies. In our study, $14 \%$ of the potential participants were not able to give written informed 
Table 1 Summary of patient characteristics and endpoints in control and intervention group

\begin{tabular}{|c|c|c|c|c|}
\hline & Item & $\begin{array}{l}\text { Control group } \\
(n=29)\end{array}$ & $\begin{array}{l}\text { Intervention } \\
\text { group }(n=31)\end{array}$ & $p$-Value \\
\hline \multirow[t]{5}{*}{ Patient characteristics } & Gender (female, \%) & $17(58.6 \%)$ & $19(61.3 \%)$ & 1.000 \\
\hline & Living situation (home-cared, \%) & $22(75 \%)$ & $20(64.5 \%)$ & 0.4051 \\
\hline & Age (mean, SD, range; years) & $\begin{array}{l}79.5 \pm 8.62 \\
(66-99)\end{array}$ & $\begin{array}{l}75.9 \pm 6.87 \\
(66-91)\end{array}$ & 0.0856 \\
\hline & Length of stay on ward (mean, SD, range; days) & $\begin{array}{l}10.1 \pm 6.0 \\
(3-29)\end{array}$ & $\begin{array}{l}11.9 \pm 8.5 \\
(4-40)\end{array}$ & 0.0671 \\
\hline & Number of drugs during hospital stay (mean, SD, range) & $\begin{array}{l}16.3 \pm 6.3 \\
(7-32)\end{array}$ & $\begin{array}{l}17.3 \pm 7.3 \\
(6-40)\end{array}$ & 0.5649 \\
\hline \multirow[t]{2}{*}{ Drug-related readmissions (DRRs) } & Number & 7 & 6 & \\
\hline & Number of patients with DRR & 7 & 3 & \\
\hline \multirow{3}{*}{$\begin{array}{l}\text { Adverse drug reactions (ADRs) } \\
\text { during follow-up }\end{array}$} & Number & 14 & 21 & \\
\hline & $\begin{array}{l}\text { Number of preventable/ameliorable ADR (NCC-MERP } \\
\text { category F-H) regarded as "preventable DRR" }\end{array}$ & 4 & 1 & \\
\hline & $\begin{array}{l}\text { Number of preventable/ameliorable ADR (NCC-MERP } \\
\text { category D-E) }\end{array}$ & 2 & 7 & \\
\hline Adverse drug reactions (ADRs) during hospital stay & Number & 9 & 9 & \\
\hline Potential inappropriate medication (PIM) & One-year prevalence of PIM & 20 & 31 & \\
\hline $\begin{array}{l}\text { Changes in medication after discharge during } \\
\text { follow-up }\end{array}$ & Number per patient & $16 \pm 11(3-47)$ & $16 \pm 10(3-52)$ & \\
\hline
\end{tabular}

SD Standard deviation

consent themselves. In addition, no legal representative was available. These circumstances make it more difficult to conduct randomized-controlled trials in this patient group. Approximately $20 \%$ of the possible participants were discharged before they could be informed about the study.

To obtain the most reliable results from this study group, a risk factor analysis was performed. In the risk factor analysis, age, the length of hospital stay and the number of changes in medication after discharge were found to have significant influence on DRRs, which is in accordance with other studies for ADRs and drugrelated hospital admissions $[19,31]$. When adding age or the length of stay to the Cox proportional hazard analysis, the effect of pharmaceutical care was statistically significant. Thus, this analysis underlines the results of previous

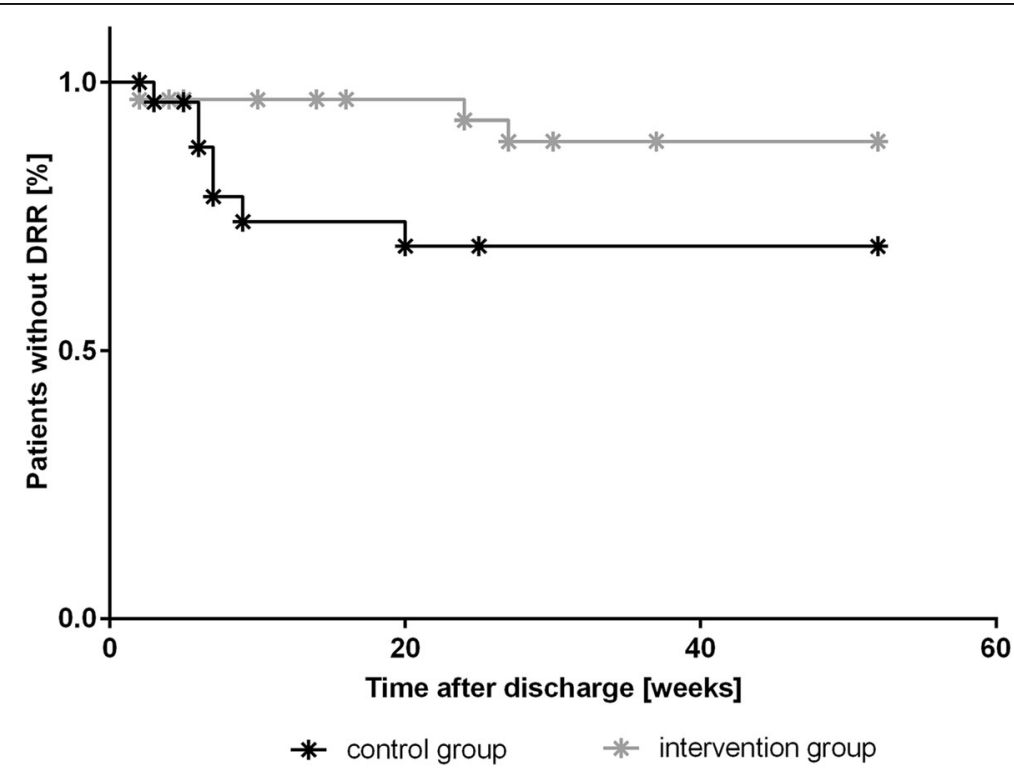

Fig. 2 Kaplan-Meier plots for the time-dependent occurrence of drug-related readmissions (DRR). Censored data are marked as * 
Table 2 Covariate analysis in a Cox-proportional hazard model. Univariate analysis was performed for each covariate

\begin{tabular}{|c|c|c|c|c|}
\hline \multirow[t]{2}{*}{ Covariate } & \multicolumn{2}{|c|}{ Effect risk factor on DRR } & \multicolumn{2}{|c|}{ Effect treatment group on DRR } \\
\hline & $\mathrm{HR}_{\mathrm{RF}}[\mathrm{Cl}]$ & $P$ value & $\mathrm{HR}_{\mathrm{TG}}[\mathrm{Cl}]$ & $P$ value \\
\hline Age [years] & $0.86[0.76-0.97]$ & $0.014^{*}$ & $4.62[1.18-18.13]$ & $0.028^{*}$ \\
\hline Gender & $0.81[0.23-2.79]$ & 0.732 & $3.26[0.84-12.66]$ & 0.088 \\
\hline Living situation & $1.01[0.21-4.94]$ & 0.989 & $3.28[0.82-13.09]$ & 0.092 \\
\hline Length of stay on ward [d] & $1.10[1.01-1.19]$ & $0.020^{*}$ & $5.76[1.15-28.85]$ & $0.033^{*}$ \\
\hline Number of PIM drugs & $0.98[0.34-2.80]$ & 0.966 & $3.26[0.83-12.81]$ & 0.090 \\
\hline Number of changes in medication after discharge & $1.06[1.01-1.11]$ & $0.013^{*}$ & $2.51[0.62-10.17]$ & 0.196 \\
\hline Number of drugs (during stay on the ward) & $1.04[0.95-1.14]$ & 0.387 & $3.54[0.90-13.89]$ & 0.070 \\
\hline
\end{tabular}

The hazard ratio (HR) describes the influence of the risk factor on the risk for DRR ("effect risk factor") or the risk for a DRR in the control group compared to the intervention group ("effect treatment group")

$R F$ risk factor, $T G$ treatment group, $C$ confidence interval, $P I M$ potentially inadequate medication

${ }^{*} P<0.05$

studies in patients with long hospital stays [21, 32]. In contrast to some studies, the younger patients were more vulnerable for DRR than the older patients in this patient population $[19,20]$. Some other studies did not identify 'age' as a risk factor for ADRs and DRRs [21,32-34].

A positive impact on preventable ADRs and medicationrelated emergency department visits, medication-related hospital admissions and readmissions in different patient populations has also been reported in previous studies $[12-14,35]$. This current study is the first to suggest a positive effect of pharmaceutical care for dependentliving elderly in particular. Moreover, the results suggest a time-dependent effect of ward-based pharmaceutical care services. After discharge, the patients had the greatest benefit from the previously performed ward-based pharmaceutical care during the first ten weeks. It seems reasonable that ward-based pharmaceutical care can influence the first weeks after discharge at most, which implies that pharmaceutical care services are also needed in ambulatory care to optimize medication of those patients continuously and to prevent ADRs and DRRs. In our study, we detected 35 ADRs in 60 patients. Thirteen of these ADRs led to drug-related readmissions in ten patients, causing a mean hospital stay of 19.8 days in this group. One patient was readmitted four times due to ADRs. Fourteen ADRs were regarded as ameliorable or preventable. Five of them caused hospital stays averaging 13.4 days. From an economic perspective, these costs are preventable for the health care system. Thus, effective methods to reduce ADRs and DRRs are needed to address the patients' and health-care system needs.

The absolute number of ADR was higher in the intervention group than in the control group. Perhaps, the intensive medication review by the pharmacist gave more signals for the researcher to detect ADR-suspicious symptoms.

Due to the limited number of participants, the efficacy of the intervention could not be confirmed in this study.
Nevertheless, the results show the potential of pharmaceutical care to influence patient-relevant endpoints and provide the basis to plan future studies in a more feasible manner, including our experience that multimorbidity and health restrictions may compromise the participation rate in the target population.

\section{Strengths}

The study was designed as randomized-controlled trial with a long follow-up of 12 months to address the need of high-quality and long follow-up trials, as stated in a Cochrane Review [6]. Parallel-group design was preferred to minimize a possible time-dependent bias. To ensure equality of observation for both treatment groups, the intervention pharmacists (CG, $\mathrm{KS}, \mathrm{NH}$ ) worked independently from the researcher (RL).

The results of this study show the potential efficacy of the intervention in a daily practice setting. The intervention was conducted as intended by the study protocol. The intervention pharmacists found 2.6 DRPs per patient on average, which aligns with the results of our previous study [20]. However, five patients received no intervention because their discharge occurred in such a narrow time frame that the intervention could not be performed.

\section{Limitations}

The small number of participants resulted in an underpowered study. Thus, statistical significance could only be shown after including covariates in the model.

As in many other studies, the Hawthorne effect could have influenced the behaviour of physicians, nurses, patients and relatives. The long follow-up may have induced a recall bias, as patients or contact persons might not have remembered less severe events that happened long ago. Additionally, a detection bias may have influenced the results, as the follow-up interviews were not blinded. We tried to avoid this bias using standardized 
questions. Furthermore, there may be a risk of subjectivity in the outcome assessment; this risk was minimized by having more than one reviewer.

Our study may have limited generalizability to nonacademic hospitals and other university hospitals because of the mono-centric study design, the selection of cooperating wards and the low participation rate. In contrast to other studies, however, we included patients of different medical disciplines representing a broader patient population [12-14].

All patients had different general practitioners, physicians, community pharmacists and other healthcare professionals who were involved in therapy and might have influenced their medication or their risk for rehospitalization. In future studies, these confounders could be documented in a structured form and might be added to the risk factor analysis.

\section{Conclusions}

This study shows the potential of a comprehensive pharmaceutical care service to reduce DRRs after discharge for elderly, cared patients in dependent-living situations. Thus, the results suggest a preliminary efficacy signal for the intervention and provide effect size estimates for powering definitive trials of this intervention in a larger patient population.

\section{Appendix 1}

Table 3 Number of participating patients in each department

\begin{tabular}{lll}
\hline Department & Control group & Intervention group \\
\hline Department of Urology & 2 & 2 \\
Department of Neurology & 10 & 18 \\
Department of Internal Medicine III & 15 & 8 \\
Department of Internal Medicine & 2 & 3 \\
Total & 29 & 31 \\
\hline
\end{tabular}

\section{Appendix 2}

Table 4 Censoring during the study follow-up

\begin{tabular}{llll}
\hline $\begin{array}{l}\text { Time of } \\
\text { contact }\end{array}$ & $\begin{array}{l}\text { Number of } \\
\text { patients in total }\end{array}$ & $\begin{array}{l}\text { Number of } \\
\text { censoring in } \\
\text { control group }\end{array}$ & $\begin{array}{l}\text { Number of } \\
\text { censoring in } \\
\text { intervention group }\end{array}$ \\
\hline $\begin{array}{l}\text { At discharge } \\
\begin{array}{l}1 \text { week } \\
\text { after discharge }\end{array}\end{array}$ & 60 & 0 & 0 \\
$\begin{array}{l}8 \text { weeks } \\
\text { after discharge }\end{array}$ & 51 & 0 & 0 \\
$\begin{array}{l}26 \text { weeks } \\
\text { after discharge }\end{array}$ & 45 & 7 & 2 \\
$\begin{array}{l}52 \text { weeks } \\
\text { after discharge }\end{array}$ & 40 & 3 & 3 \\
\hline
\end{tabular}

\section{Appendix 3}

Table 5 DRP categories of the intervention group ( $n=26$ patients)

\begin{tabular}{ll}
\hline DRP category & Number of DRP \\
\hline Drug & 10 \\
Dosage form/drug strength & 1 \\
Dosage & 7 \\
Indication & 14 \\
Contraindication & 3 \\
Drug-drug interaction & 36 \\
Adverse drug reaction & 5 \\
Administration/adherence & 5 \\
Administration & 5 \\
Other & 14 \\
Total & 100 \\
\hline
\end{tabular}

\section{Abbreviations}

ADR: Adverse drug reaction; CG: Control group; CRF: Case report form: DRP: Drug-related problem; DRR: Drug-related readmission; HR: Hazard ratio; IG: Intervention group; PIM: Potential inappropriate medication

\section{Acknowledgements}

We gratefully thank the pharmacists Nicole Hohn (NH), Theresa Hermanspann, Claudia Bollig and Julia Amkreutz for their outstanding support of this study. We thank all patients participating in this project, the health-care teams on the wards for their support, Hanna M. Seidling, Luigi Villa and Linda Krolop for their engaged participation in the expert panel, and Niklas Lenssen for carefully reading and correcting the manuscript.

\section{Funding}

This work was supported by the Foerderinitiative Pharmazeutische Betreuung e.V. and the Apothekerstiftung Nordrhein. RL received a research grant from the "Foerderinitiative Pharmazeutische Betreuung e.V.". RL, UJ and AE received a research grant from the Apotheker-Stiftung Nordrhein. The funding bodies had no influence on the design of the study, data collection, analysis and interpretation, and writing the manuscript.

Availability of data and materials

The datasets generated and/or analysed during the current study are not publicly available. Anonymized datasets are available from the corresponding author upon reasonable request.

Authors' contributions

$\mathrm{RL}, \mathrm{AH}, \mathrm{JBS}, \mathrm{CT}, \mathrm{NM}, \mathrm{CF}, \mathrm{UJ}$, AE: study concept and design. RL, KS, CG: data acquisition. RL, UJ, CF: data analysis and interpretation. All authors contributed to drafting or revising the article critically for intellectual content. All authors read and approved the final version of the manuscript.

\section{Ethics approval and consent to participate}

The study was approved by the local ethics committee (ethic committee RWTH Aachen University, EK 195/12). Written informed consent was obtained from all individual participants or their legal representatives.

\section{Competing interests}

The authors declare that they have no competing interests.

\section{Publisher's Note}

Springer Nature remains neutral with regard to jurisdictional claims in published maps and institutional affiliations. 


\section{Author details}

'Hospital Pharmacy, University Hospital RWTH Aachen, Steinbergweg 20, 52074 Aachen, Germany. ${ }^{2}$ Department of Urology, University Hospital RWTH Aachen, Pauwelsstr. 30, 52074 Aachen, Germany. ${ }^{3}$ Department of Neurology, University Hospital RWTH Aachen, Pauwelsstr. 30, 52074 Aachen, Germany. ${ }^{4}$ Department of Internal Medicine III, Gastroenterology, Metabolic Disorders and Internal Intensive Medicine, University Hospital RWTH Aachen, Pauwelsstr. 30, 52074 Aachen, Germany. ${ }^{5}$ Department of Internal Medicine I, Cardiology, Pneumology, Angiology and Internal Intensive Medicine, University Hospital RWTH Aachen, Pauwelsstr. 30, 52074 Aachen, Germany. ${ }^{6}$ Department of Medical Statistics, RWTH Aachen University, Pauwelsstr. 30, 52074 Aachen, Germany. ${ }^{7}$ Institute of Pharmacy, Clinical Pharmacy, University of Bonn, An der Immenburg 4, 53121 Bonn, Germany. ${ }^{8}$ Present address: Hospital Pharmacy, University Hospital Cologne, Kerpener Str. 62, 50937 Cologne, Germany. ${ }^{9}$ Present address: Department of Urology, University Hospital Cologne, Kerpener Str. 62, 50937 Cologne, Germany.

Received: 30 August 2017 Accepted: 9 May 2018

Published online: 04 June 2018

\section{References}

1. Forster AJ, Clark HD, Menard A, Dupuis N, Chernish R, Chandok N, et al. Adverse events among medical patients after discharge from hospital. CMAJ. 2004:170:345-9.

2. Tache SV, Sonnichsen A, Ashcroft DM. Prevalence of adverse drug events in ambulatory care: a systematic review. Ann Pharmacother. 2011:45:977-89.

3. Chan M, Nicklason F, Vial JH. Adverse drug events as a cause of hospital admission in the elderly. Intern Med J. 2001;31:199-205.

4. Laroche ML, Charmes JP, Nouaille Y, Picard N, Merle L. Is inappropriate medication use a major cause of adverse drug reactions in the elderly? $\mathrm{Br} J$ Clin Pharmacol. 2007;63:177-86.

5. Beard K. Adverse reactions as a cause of hospital admission in the aged. Drugs Aging. 1992;2:356-67.

6. Christensen M, Lundh A. Medication review in hospitalised patients to reduce morbidity and mortality. Cochrane Database Syst Rev. 2016;2:Cd008986.

7. Rennke S, Shoeb MH, Nguyen OK, Magan Y, Wachter RM, Ranji SR. Interventions to improve care transitions at hospital discharge. In: Agency for Healthcare Research and Quality (US); Rockville M, Editor. Making Health Care Safer II: An Updated Critical Analysis of the Evidence for Patient Safety Practices, 2013. https://www.ahrq.gov/sites/default/files/wysiwyg/research/ findings/evidence-based-reports/services/quality/patientsftyupdate/ ptsafetyll-full.pdf. Accessed 16 May 2018.

8. Brilli RJ, Spevetz A, Branson RD, Campbell GM, Cohen H, Dasta JF, et al. Critical care delivery in the intensive care unit: defining clinical roles and the best practice model. Crit Care Med. 2001;29:2007-19.

9. Preslaski CR, Lat I, MacLaren R, Poston J. Pharmacist contributions as members of the multidisciplinary ICU team. Chest. 2013;144:1687-95.

10. Jorch G, Kluge S, König F, Markewitz A, Notz K, Parvu V et al. Empfehlungen zur Struktur und Ausstattung von Intensivstationen. https://www.divi.de/ empfehlungen/publikationen/intensivmedizin/399-empfehlungen-zurstruktur-von-intensivstationen-langversion/file. Accessed 16 May 2018.

11. Seidling $H$, Lenssen R. Empfehlungen zur Arzneimitteltherapiesicherheit im Krankenhaus. Z Evid Fortbild Qual Gesundhwes. 2014;108:44-8.

12. Schnipper JL, Kirwin JL, Cotugno MC, Wahlstrom SA, Brown BA, Tarvin E, et al. Role of pharmacist counseling in preventing adverse drug events after hospitalization. Arch Intern Med. 2006;166:565-71.

13. Gillespie U, Alassaad A, Henrohn D, Garmo H, Hammarlund-Udenaes M, Toss $\mathrm{H}$, et al. A comprehensive pharmacist intervention to reduce morbidity in patients 80 years or older: a randomized controlled trial. Arch Intern Med. 2009;169:894-900.

14. Hellstrom LM, Bondesson A, Hoglund P, Midlov P, Holmdahl L, Rickhag E, et al. Impact of the Lund integrated medicines management (LIMM) model on medication appropriateness and drug-related hospital revisits. Eur J Clin Pharmacol. 2011;67:741-52

15. Gallagher J, Byrne S, Woods N, Lynch D, McCarthy S. Cost-outcome description of clinical pharmacist interventions in a university teaching hospital. BMC Health Serv Res. 2014;14:177.

16. De Rijdt T, Willems $L$, Simoens S. Economic effects of clinical pharmacy interventions: a literature review. Am J Health Syst Pharm. 2008;65:1161-72.

17. Scullin C, Scott MG, Hogg A, McElnay JC. An innovative approach to integrated medicines management. J Eval Clin Pract. 2007;13:781-8.
18. Gustafsson M, Sjolander M, Pfister B, Jonsson J, Schneede J, Lovheim H. Pharmacist participation in hospital ward teams and hospital readmission rates among people with dementia: a randomized controlled trial. Eur J Clin Pharm. 2017;73:827-35.

19. Leendertse AJ, Egberts AC, Stoker LJ, van den Bemt PM. Frequency of and risk factors for preventable medication-related hospital admissions in the Netherlands. Arch Intern Med. 2008;168:1890-6.

20. Lenssen R, Heidenreich A, Schulz JB, Trautwein C, Fitzner C, Jaehde U, et al. Analysis of drug-related problems in three departments of a German University hospital. Int J Clin Pharm. 2016;38:119-26.

21. Garcia-Perez L, Linertova R, Lorenzo-Riera A, Vazquez-Diaz JR, DuqueGonzalez B, Sarria-Santamera A. Risk factors for hospital readmissions in elderly patients: a systematic review. OJM. 2011:104:639-51.

22. Hohmann C, Eickhoff C, Klotz JM, Schulz M, Radziwill R. Development of a classification system for drug-related problems in the hospital setting (APS-doc) and assessment of the inter- rater reliability. J Clin Pharm Ther. 2012;37:276-81.

23. Arimone $Y$, Miremont-Salame G, Haramburu F, Molimard M, Moore $N_{\text {, }}$ Fourrier-Reglat A, et al. Inter-expert agreement of seven criteria in causality assessment of adverse drug reactions. Br J Clin Pharmacol. 2007;64:482-8.

24. Schumock GT, Thornton JP. Focusing on the preventability of adverse drug reactions. Hosp Pharm. 1992;27:538.

25. Snyder RA, Abarca J, Meza JL, Rothschild JM, Rizos A, Bates DW. Reliability evaluation of the adapted national coordinating council medication error reporting and prevention (NCC MERP) index. Pharmacoepid Drug Saf. 2007; 16:1006-13

26. Koordinierungsgruppe zur Umsetzung und Fortschreibung des Aktionsplans des Bundesministeriums für Gesundheit zur Verbesserung der Arzneimitteltherapiesicherheit in Deutschland (Aktionsplan AMTS). Definitionen zur Pharmakovigilanz und Arzneimitteltherapiesicherheit (AMTS). Krankenhauspharmazie. 2014;35:425-8.

27. Holt S, Schmiedl S, Thurmann PA. Potentially inappropriate medications in the elderly: the PRISCUS list. Dtsch Arztebl Int. 2010;107:543-51.

28. Foundation Pharmaceutical Care Network Europe. The PCNE Classification V 6.2. 2010. http://www.pcne.org/upload/files/11_PCNE_classification_V6-2.pdf Accessed 15 Jan 2018

29. Zwiener I, Blettner M, Hommel G. Survival analysis: part 15 of a series on evaluation of scientific publications. Dtsch Arztebl Int. 2011:108:163-9.

30. Petty DR, Zermansky AG, Raynor DK, Vail A, Lowe CJ, Freemantle N, et al. "No thank you": why elderly patients declined to participate in a research study. Pharm World Sci. 2001;23:22-7.

31. Zhang $\mathrm{M}$, Holman $\mathrm{CD}$, Preen $\mathrm{DB}$, Brameld K. Repeat adverse drug reactions causing hospitalization in older Australians: a population-based longitudinal study 1980-2003. Br J Clin Pharmacol. 2007;63:163-70.

32. Gray SL, Sager M, Lestico MR, Jalaluddin M. Adverse drug events in hospitalized elderly. J Gerontol A Biol Sci Med Sci. 1998;53:M59-63.

33. Zhang M, Holman CD, Price SD, Sanfilippo FM, Preen DB, Bulsara MK Comorbidity and repeat admission to hospital for adverse drug reactions in older adults: retrospective cohort study. BMJ. 2009:338:a2752.

34. Campbell SE, Seymour DG, Primrose WR. A systematic literature review of factors affecting outcome in older medical patients admitted to hospital. Age Ageing. 2004;33:110-5.

35. Leendertse AJ, de Koning GH, Goudswaard AN, Belitser SV, Verhoef M, de Gier $\mathrm{HJ}$, et al. Preventing hospital admissions by reviewing medication (PHARM) in primary care: an open controlled study in an elderly population. I Clin Pharm Ther. 2013;38:379-87.

\section{Ready to submit your research? Choose BMC and benefit from}

- fast, convenient online submission

- thorough peer review by experienced researchers in your field

- rapid publication on acceptance

- support for research data, including large and complex data types

- gold Open Access which fosters wider collaboration and increased citations

- maximum visibility for your research: over $100 \mathrm{M}$ website views per year

\section{At BMC, research is always in progress.}

Learn more biomedcentral.com/submissions 